\title{
New iron tetrazolate frameworks: synthesis temperature effect, thermal behavior, Mössbauer and magnetic studies
}

Vanessa Pimenta, ${ }^{a}$ Quang Hoang Hanh Le, ${ }^{\text {a }}$ Lucy Clark, ${ }^{b}$ Jérôme Lhoste, ${ }^{\text {a, }}$ Annie HémonRibaud, ${ }^{\mathrm{a}}$ Marc Leblanc, ${ }^{\mathrm{a}}$ Jean-Marc Grenèche, ${ }^{\mathrm{a}}$ Gilles Dujardin, ${ }^{\mathrm{a}}$ Philip Lightfoot ${ }^{\mathrm{b}}$ and Vincent Maisonneuve. ${ }^{\mathrm{a}}$

${ }^{a}$ IMMM-UMR 6283 CNRS, LUNAM, Faculté des Sciences et Techniques, Université du Maine, Avenue Olivier Messiaen, 72085 Le Mans Cedex 9, France

${ }^{b}$ School of Chemistry and EaStChem, University of St. Andrews, St. Andrews, Fife, KY16 9ST, United Kingdom

* To whom correspondence should be addressed. E-mail: jerome.lhoste@univ-lemans.fr Phone: (33).(0)2 438333 59. Fax: (33).(0)2 43833506 
Table S1. Atomic coordinates and equivalent ADP of $[\mathrm{Hdma}] \cdot\left(\mathrm{Fe}_{5} \mathrm{~F}_{8}\left(\mathrm{H}_{2} \mathrm{O}\right)_{2}(\text { amtetraz })_{4}\right)(1)$

\begin{tabular}{|c|c|c|c|c|c|c|}
\hline Atom & $\begin{array}{c}\text { Wyckoff } \\
\text { position }\end{array}$ & $\tau$ & $\mathbf{x}$ & $\mathbf{y}$ & $\mathbf{z}$ & $\mathbf{B}_{\text {eq }}\left(\AA^{2}\right)$ \\
\hline $\mathrm{Fe}(1)$ & 1d & 1 & $1 / 2$ & 0 & 0 & $3,34(8)$ \\
\hline $\mathrm{Fe}(2)$ & $1 \mathrm{a}$ & 1 & 0 & 0 & 0 & $3,24(8)$ \\
\hline $\mathrm{Fe}(3)$ & $2 \mathrm{l}$ & 1 & $1 / 2$ & $0.36593(9)$ & $1 / 2$ & $1,83(3)$ \\
\hline $\mathrm{Fe}(4)$ & $1 \mathrm{f}$ & 1 & 0 & $1 / 2$ & $1 / 2$ & $2,16(5)$ \\
\hline $\mathrm{F}(1)$ & $2 \mathrm{~m}$ & 1 & $0.190(2)$ & 0 & $-0.1361(7)$ & $3,1(2)$ \\
\hline $\mathrm{F}(2)$ & $4 \mathrm{o}$ & 1 & $0.2041(9)$ & $0.3842(4)$ & $0.5206(12)$ & $5,1(2)$ \\
\hline $\mathrm{F}(3) / \mathrm{OW}(3)$ & $2 \mathrm{n}$ & $0.5 / 0.5$ & $0.587(2)$ & $1 / 2$ & $0.6606(9)$ & $3,5(2)$ \\
\hline $\mathrm{F}(4) / \mathrm{OW}(4)$ & $2 \mathrm{n}$ & $0.5 / 0.5$ & $0.044(2)$ & $1 / 2$ & $0.7241(12)$ & $5,9(4)$ \\
\hline $\mathrm{N}(1)$ & $4 \mathrm{o}$ & 1 & $0.209(2)$ & $0.1248(7)$ & $0.1406(12)$ & $4,3(4)$ \\
\hline $\mathrm{N}(2)$ & $4 \mathrm{o}$ & 1 & $0.418(2)$ & $0.1234(7)$ & $0.1468(10)$ & $4,7(3)$ \\
\hline $\mathrm{N}(3)$ & $4 \mathrm{o}$ & 1 & $0.522(2)$ & $0.1964(7)$ & $0.2489(11)$ & $4,3(3)$ \\
\hline $\mathrm{N}(4)$ & $4 \mathrm{o}$ & 1 & $0.399(2)$ & $0.2479(4)$ & $0.3127(8)$ & $3,6(2)$ \\
\hline $\mathrm{N}(5)$ & $4 \mathrm{o}$ & 1 & $-0.0029(16)$ & $0.2336(8)$ & $0.2700(16)$ & $5,6(3)$ \\
\hline $\mathrm{C}(1)$ & $4 \mathrm{o}$ & 1 & $0.204(2)$ & $0.2018(8)$ & $0.2425(12)$ & $3,9(3)$ \\
\hline $\mathrm{C}(1) \mathrm{A}$ & $2 \mathrm{j}$ & 0.5 & $1 / 2$ & $0.401(3)$ & 0 & $3,5(6)$ \\
\hline $\mathrm{N}(1) \mathrm{A}$ & $2 \mathrm{n}$ & 0.25 & $0.457(8)$ & $1 / 2$ & $-0.107(6)$ & $2,8(9)$ \\
\hline $\mathrm{C}(2) \mathrm{A}$ & $2 \mathrm{~m}$ & 0.50 & $0.185(3)$ & 0 & $-0.503(4)$ & $2,1(3)$ \\
\hline $\mathrm{N}(2) \mathrm{A}$ & $4 \mathrm{o}$ & 0.125 & $0.003(11)$ & $0.044(4)$ & $-0.446(6)$ & $2,4(9)$ \\
\hline
\end{tabular}

Table S2. Atomic coordinates and equivalent ADP of $[\mathrm{Hdma}]_{1.5} \cdot\left(\mathrm{Fe}_{5} \mathrm{~F}_{7}\left(\mathrm{H}_{2} \mathrm{O}\right)(\mathrm{HCOO})(\mathrm{amtetraz})_{4}\right)(2)$

\begin{tabular}{|c|c|c|c|c|c|c|}
\hline Atom & $\begin{array}{c}\text { Wyckoff } \\
\text { position }\end{array}$ & $\tau$ & $\mathbf{x}$ & $\mathbf{y}$ & $\mathbf{z}$ & $\mathbf{B}_{\text {eq }}\left(\AA^{2}\right)$ \\
\hline $\mathrm{Fe}(1)$ & $1 \mathrm{~d}$ & 1 & $1 / 2$ & 0 & 0 & $1,24(2)$ \\
\hline $\mathrm{Fe}(2)$ & $1 \mathrm{a}$ & 1 & 0 & 0 & 0 & $1,11(2)$ \\
\hline $\mathrm{Fe}(3)$ & $2 \mathrm{l}$ & 1 & $1 / 2$ & $0.36653(6)$ & $1 / 2$ & $1,29(2)$ \\
\hline $\mathrm{Fe}(4)$ & $1 \mathrm{f}$ & 1 & 0 & $1 / 2$ & $1 / 2$ & $1,46(2)$ \\
\hline $\mathrm{F}(1)$ & $2 \mathrm{~m}$ & 1 & $0.8042(5)$ & 0 & $0.1388(4)$ & $1,76(6)$ \\
\hline $\mathrm{F}(2)$ & $4 \mathrm{o}$ & 1 & $0.2183(4)$ & $0.38055(18)$ & $0.5425(3)$ & $2,16(4)$ \\
\hline $\mathrm{F}(3) / \mathrm{OW}(3)$ & $2 \mathrm{n}$ & $0.25 / 0.75$ & $0.6061(6)$ & $1 / 2$ & $0.6660(5)$ & $2,03(6)$ \\
\hline $\mathrm{F}(4) / \mathrm{OW}(4)$ & $2 \mathrm{n}$ & $0.25 / 0.75$ & $-0.0035(8)$ & $1 / 2$ & $0.2577(5)$ & $3,53(9)$ \\
\hline $\mathrm{N}(1)$ & $4 \mathrm{o}$ & 1 & $0.7972(5)$ & $0.1250(3)$ & $0.8589(4)$ & $1,64(6)$ \\
\hline $\mathrm{N}(2)$ & $4 \mathrm{o}$ & 1 & $0.5890(5)$ & $0.1212(3)$ & $0.8501(4)$ & $1,65(6)$ \\
\hline $\mathrm{N}(3)$ & $4 \mathrm{o}$ & 1 & $0.4812(5)$ & $0.1927(3)$ & $0.7506(4)$ & $1,84(6)$ \\
\hline $\mathrm{N}(4)$ & $4 \mathrm{o}$ & 1 & $0.6162(5)$ & $0.2471(3)$ & $0.6892(4)$ & $1,60(6)$ \\
\hline $\mathrm{C}(1)$ & $4 \mathrm{o}$ & 1 & $0.8082(6)$ & $0.2050(3)$ & $0.7593(5)$ & $1,76(6)$ \\
\hline $\mathrm{N}(5)$ & $4 \mathrm{o}$ & 1 & $0.9897(6)$ & $0.2360(4)$ & $0.7306(6)$ & $4,1(2)$ \\
\hline $\mathrm{C}(4)$ & $2 \mathrm{n}$ & 0.50 & $0.176(2)$ & $1 / 2$ & $0.2290(18)$ & $3,4(3)$ \\
\hline $\mathrm{C}(1) \mathrm{A}$ & $2 \mathrm{j}$ & 0.75 & $1 / 2$ & $0.4010(7)$ & 0 & $3,9(3)$ \\
\hline $\mathrm{N}(1) \mathrm{A}$ & $2 \mathrm{n}$ & 0.375 & $0.437(2)$ & $1 / 2$ & $0.9086(17)$ & $2,8(3)$ \\
\hline $\mathrm{C}(2) \mathrm{A}$ & $2 \mathrm{~m}$ & 0.75 & $-0.1875(16)$ & 0 & $0.5031(11)$ & $3,6(3)$ \\
\hline $\mathrm{N}(2) \mathrm{A}$ & $4 \mathrm{o}$ & 0.1875 & $-0.050(4)$ & $-0.0477(18)$ & $0.460(2)$ & $3,5(5)$ \\
\hline
\end{tabular}




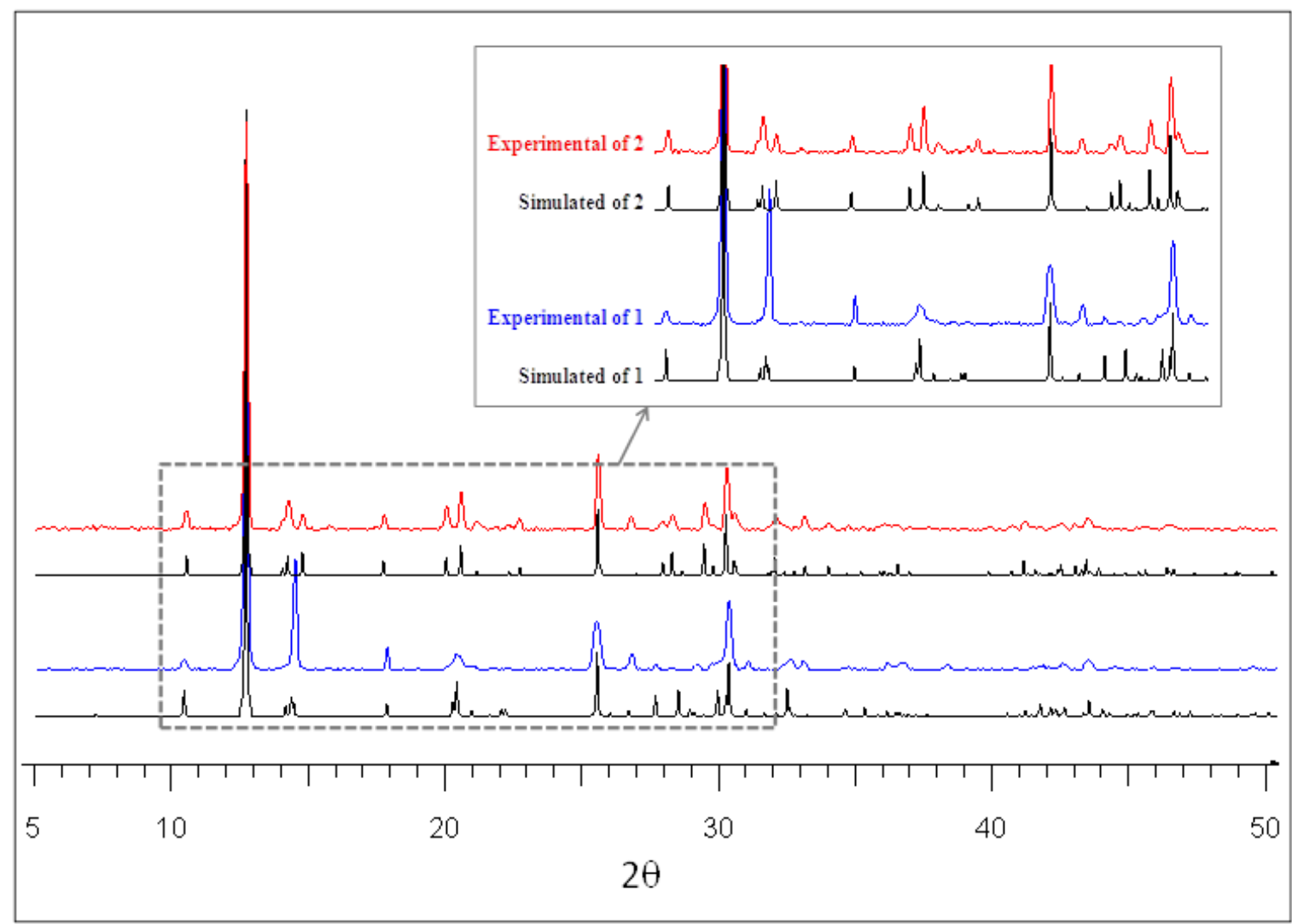

Figure S1. Experimental and simulated XRD patterns of $[\mathrm{Hdma}] \cdot\left(\mathrm{Fe}_{5} \mathrm{~F}_{8}\left(\mathrm{H}_{2} \mathrm{O}\right)_{2}(\text { amtetraz })_{4}\right)(1)$ and $[\mathrm{H} d m a]_{1.5} \cdot\left(\mathrm{Fe}_{5} \mathrm{~F}_{7}\left(\mathrm{H}_{2} \mathrm{O}\right)(\mathrm{HCOO})\left(\right.\right.$ amtetraz $\left._{4}\right)(2)$

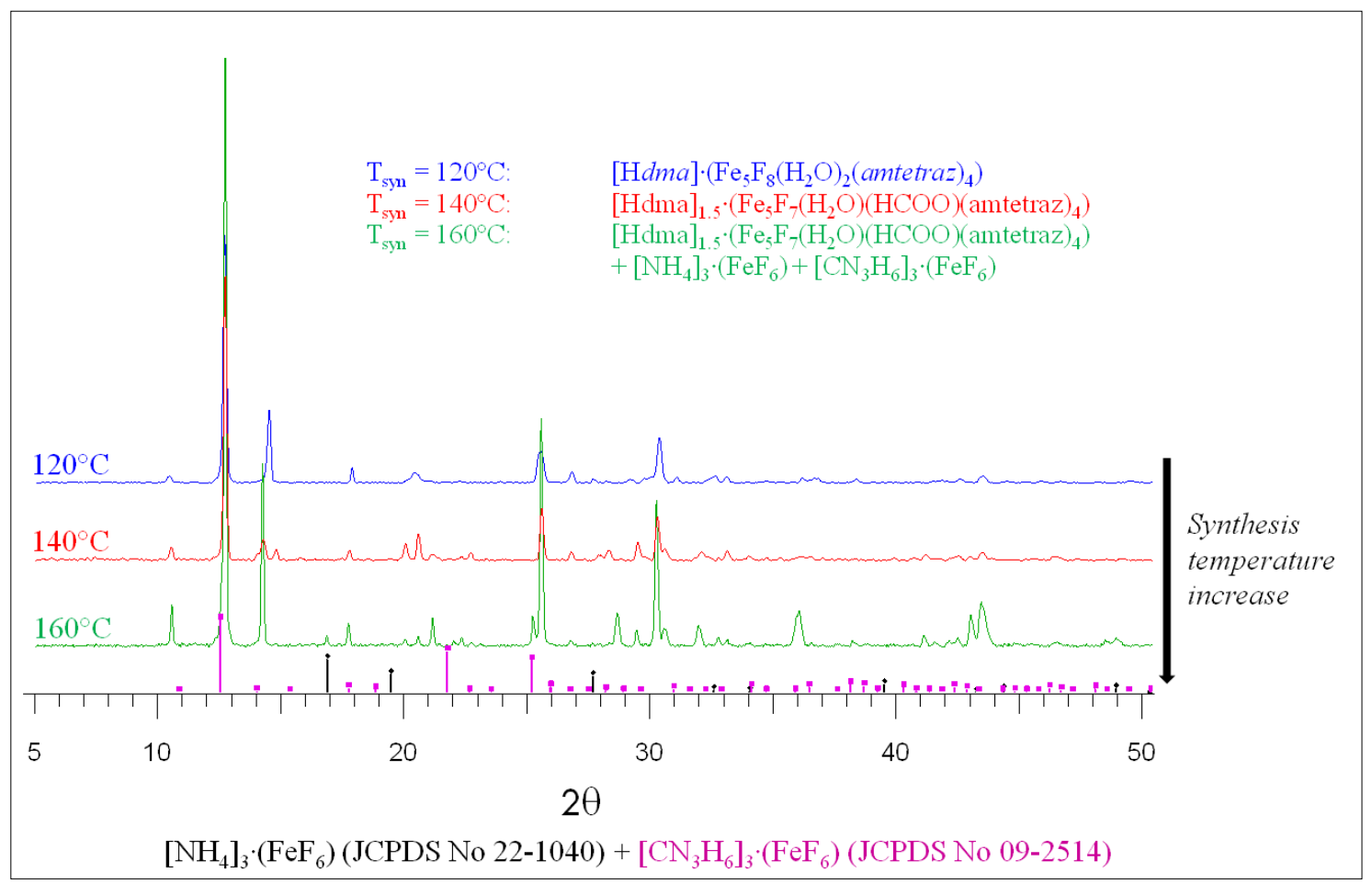

Figure S2. Evolution of XRD patterns and phase composition as a function of the synthesis temperature 


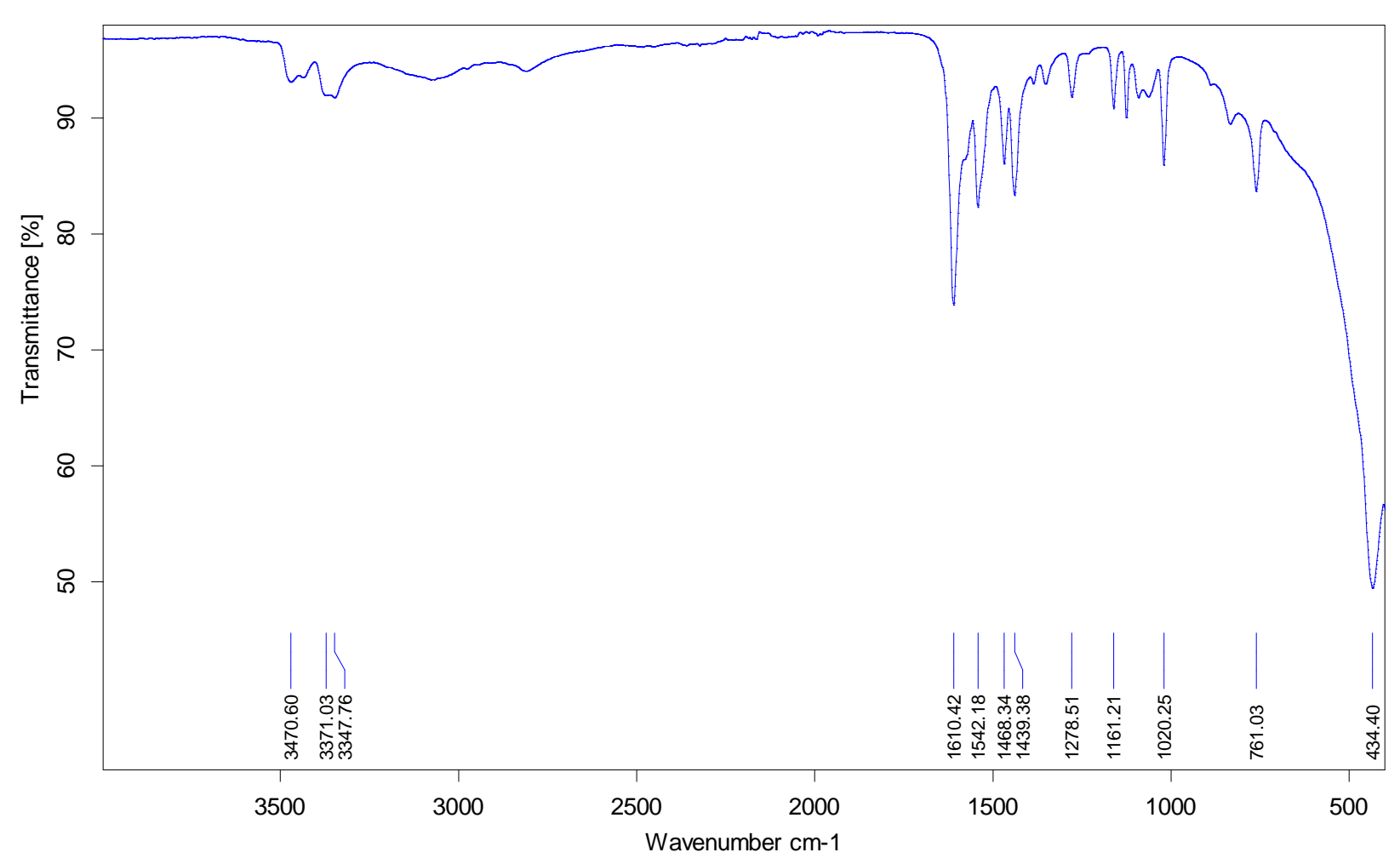

Figure S3. Infrared spectroscopy of $[\mathrm{Hdma}] \cdot\left(\mathrm{Fe}_{5} \mathrm{~F}_{8}\left(\mathrm{H}_{2} \mathrm{O}\right)_{2}(\text { amtetraz })_{4}\right)(1)$

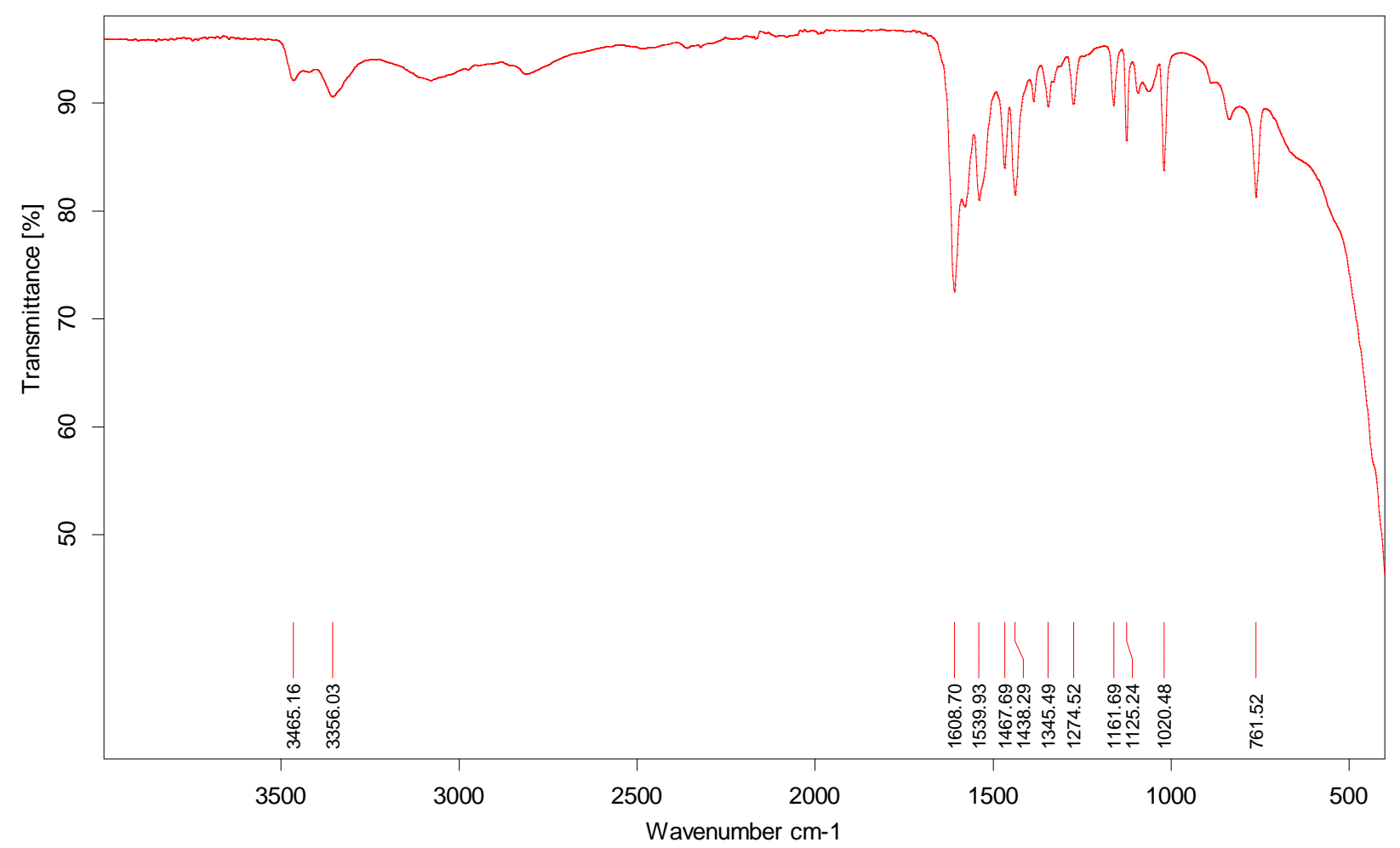

Figure S4. Infrared spectroscopy of $[\mathrm{Hdma}]_{1.5} \cdot\left(\mathrm{Fe}_{5} \mathrm{~F}_{7}\left(\mathrm{H}_{2} \mathrm{O}\right)(\mathrm{HCOO})(\text { amtetraz })_{4}\right)(2)$ 